Since the zinc ammonium phosphate is not precipitated in presence of a large excess of ammonia, the process may be used in the presence of magnesium which is precipitated in the strongly alkaline liquid, and the filtrate from the precipitate neutralized to precipitate the zinc. About I.I 5 grams of crystallized magnesium sulphate were added to some of the zinc solution, made strongly alkaline with ammonia, sodium phosphate added, and after standing about fifteen minutes with frequent stirring, filtered, washed, and the zinc determined in the filtrate.

$$
\begin{array}{cc}
\text { Zinc taken. } & \text { Zinc found. } \\
0 . \mathrm{r} 490 & 0.148 \mathrm{r}
\end{array}
$$

The process gives fairly good results in the presence of iron, calcium, and magnesium, as the following results will show. Where unknown but rather large quantities of solutions of iron (ferric), calcium, and magnesium salts were added to the zinc solution, the whole being strongly alkaline, sodium phosphate was added in large excess, the solution being in a graduated flask which was filled to the mark, mixed, filtered through dry paper and an aliquot part taken for determining the zinc.

Zinc taken.
Gram.
O.II92
o.1192

$$
\begin{aligned}
& \text { Zine found. } \\
& \text { Gram. } \\
& 0.117^{2} \\
& 0.1172
\end{aligned}
$$

Manganese, however, must be previously separated, best by the nitric acid and potassium chlorate method.

UNIVERSITY OF ARkANSAS, May 8 , $190 \mathrm{r}$.

\title{
THE EXTRACTION OF MORPHINE WITH IMMISCIBLE SOLVENTS.
}

BY W. A. PUCKXER.

Received June 11, soon.

A recent publication by $F$. Wirthle relative to the extraction of morphine from its solution by means of a chloroform alcohol mixture leads me to publish some similar determinations. My experiments were made with a view of ascertaining whether instead of liberating the alkaloid by addition of fixed alkali there might be substituted ammonium hydroxide. This work was not completed on account of similar determinations published by Kippenberger ${ }^{2}$. While I did not find the substitution of ammonia ad-

1 Chem. Ztg, 25, $29 \mathrm{~s}$.

${ }_{2}$ Ztschr. anal. Chem., 39, 290. 


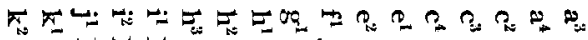

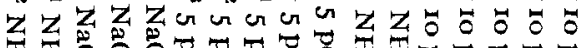

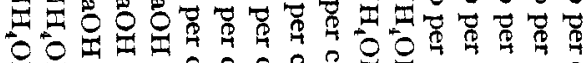
西政N

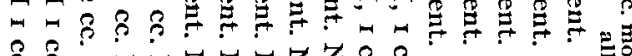

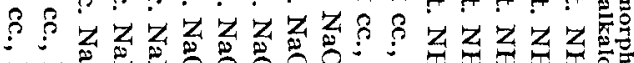

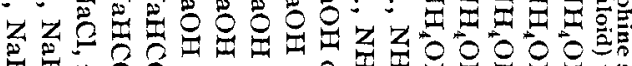

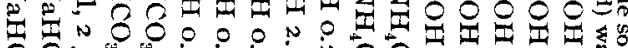

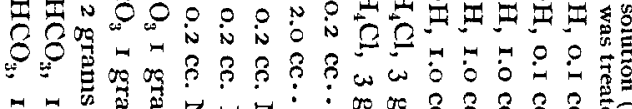
*

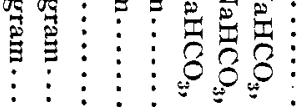

$\vdots$ 然然然

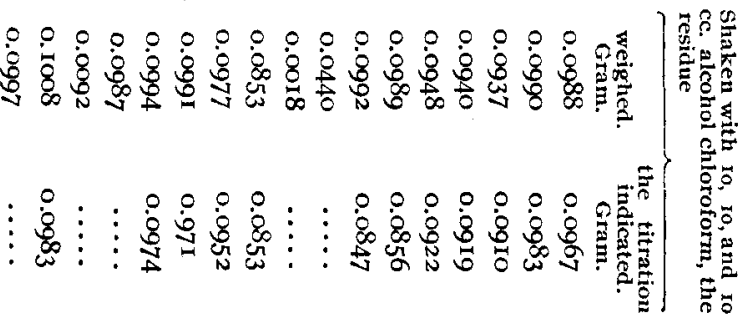

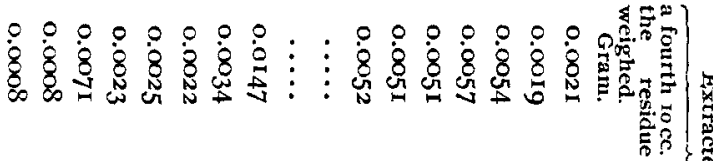

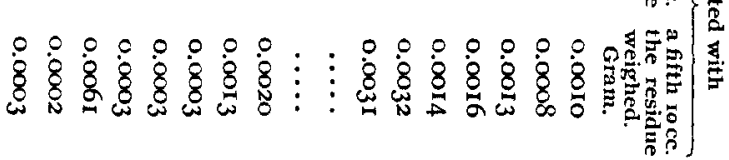

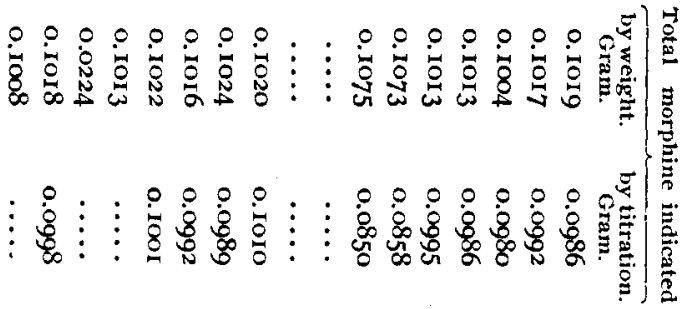


vantageous yet $\mathrm{my}$ results were more favorable than those of Wirthle and hence may be of some interest.

The morphine alkaloid used was purified by crystallization from hot absolute alcohol. Its purity, determined by titration with decinormal sulphuric acid and cochineal as indicator, was found to correspond to $99.5 \circ$ per cent. monohydrated morphine $\left(\mathrm{C}_{17} \mathrm{H}_{19} \mathrm{NO}_{3}, \mathrm{H}_{2} \mathrm{O}\right)$. Of this, 2.5093 grams, corresponding to 2.4968 grams pure morphine, were dissolved in Io cc. volumetric normal hydrochloric acid and sufficient water added to make $250 \mathrm{cc}$.

To extract and estimate the morphine in this solution Io $\mathrm{cc}$, representing 0.0998 gram morphine alkaloid, were in each case measured into a separatory funnel, rendered alkaline as below stated and then shaken with successive portions of a mixture composed of 80 volumes of chloroform and 20 volumes of alcohol. Three extractions of $\mathrm{I} O \mathrm{cc}$. each were received in a shallow beaker (A). A further extraction of Io cc. was received in a second beaker (B), and still another ro cc. received in a third beaker (C). These liquids were allowed to evaporate spontaneously, ${ }^{1}$ the residue kept over sulphuric acid for several hours, weighed, and then titrated with volumetric decinormal sulphuric acid, using cochineal as indicator.

These results show that while ammonium hydroxide combines with morphine to form a salt that is not readily taken up with the alcohol chloroform, it does so to a much smaller extent than sodium hydroxide. If as in $a$ but a slight excess of ammonia is added, approximately 98 per cent. of the alkaloid is removed by the first three extractions. Even if ten times the amount used in $a$ is added as in series $c$ three extractions still remove more than go per cent., and practically all the alkaloid is obtained when five portions of the solvent are used. If a few drops of the cochineal indicator be added to the morphine solution and ammonia added to alkalinity the quantity used in $a$ need not be exceeded. As might be expected the addition of sodium bicarbonate, $k$, overcomes the effect of an excess of ammonium hydroxide just as Kippenberger found this to be the case for fixed alkali. The

1 If the evaporation is hastened by application of heat then a vamish-like residue is obtained from which the last traces of alcohol are expelled only with much difficulty. The residue left when the liquid has evaporated spontatneously is erystalline and loses no weight when dried at $60^{\circ} \mathrm{C} .: i$. e, morphine containing one molecule of water is thus obtained. 
attempt to decrease the dissociation of the morphinate by ammonium chloride in $e$ and thus permit its removal from the solution did not prove successful. The ammonium chloride apparently acts as so much free ammonia. The discrepancy in $e$ between the weight of the residue and the morphine indicated by titration is due to ammonium chloride taken up by the solvent; to a moderate degree such disagreement is seen in all determinations.

UNIVERSITY OF ILLINOIS.

\section{IMPROVED ELECTRIC FURNACE FOR LABORATORY USE.}

By Samuel AUCemety TUCker and Herbert R. Moody.

$$
\text { Received June } 1 \text {, roor. }
$$

$E_{\text {writers to possess certain disadvantages for experimental }}^{\text {LECTRIC furnaces hitherto described have been found by the }}$ purposes. This led them to devise the form of furnace herein described which it is thought is a convenient piece of apparatus to build and will be found to be well adapted to a variety of work in the laboratory.

The Moissan type of furnace is now well known to every one, but it is troublesome in several ways. If built from chalk blocks in the rough, it takes both skill and time to cut them to the exact form required; it is then necessary to dry the blocks very thoroughly, otherwise they will crack in all directions and render the furnace useless immediately. A perfectly dry furnace supported by metallic bands will generally crack to some extent, and it is seldom possible to put the furnace in use a second time. The form of furnace described by one of $\mathrm{us}^{1}$ which simply consists of a graphite crucible forming one pole, the other being a carbon rod supported vertically with an arrangement for raising and lowering it at will is useful for some purposes, but there is too much exposure to the air for many operations, and the material of which the crucible is composed is likely to introduce undesirable impurities during the fusion. The operator is exposed to very intense radiant heat which interferes considerably with its use for any lengthened period. The present form of furnace is after the Moissan type and is composed of carbon bricks I 2 inches by 4 inches by 2 inches, luted together with Dixon stove paste. The sides of the furnace were of 6 -inch brick, thus making

1 American Electrician, $11,408$. 